\title{
DNA microarray analysis of Salmonella serotype Typhimurium strains causing different symptoms of disease
}

\author{
Eva Litrup ${ }^{1 *}$, Mia Torpdahl1', Burkhard Malorny², Stephan Huehn², Morten Helms ${ }^{3}$, Henrik Christensen ${ }^{4}$, \\ Eva M Nielsen ${ }^{1}$
}

\begin{abstract}
Background: Salmonella enterica subsp. enterica is one of the leading food-borne pathogens in the USA and European countries. Outcome of human Salmonella serotype Typhimurium infections ranges from mild self-limiting diarrhoea to severe diarrhoea that requires hospitalization. Increased knowledge of the mechanisms that are responsible for causing infection and especially the severity of infection is of high interest.

Results: Strains were selected from patients with mild infections $(n=9)$ and patients with severe infections $(n=9)$ and clinical data allowed us to correct for known underlying diseases. Additionally, outbreak isolates $(n=3)$ were selected. Strains were analyzed on a DNA-DNA microarray for presence or absence of 281 genes covering marker groups of genes related to pathogenicity, phages, antimicrobial resistance, fimbriae, mobility, serotype and metabolism. Strains showed highly similar profiles when comparing virulence associated genes, but differences between strains were detected in the prophage marker group. The Salmonella virulence plasmid was present in $72 \%$ of the strains, but presence or absence of the virulence plasmid did not correspond to disease symptoms. A dendrogram clustered strains into four groups. Clustering confirmed DT104 as being a clonal phagetype. Clustering of the remaining strains was mainly correlated to presence or absence of the virulence plasmid and mobile elements such as transposons. Each of the four clusters in the tree represented an almost equal amount of strains causing severe or mild symptoms of infection.

Conclusions: We investigated clinical significance of known virulence factors of Salmonella serotype Typhimurium strains causing different disease symptoms, and conclude that the few detected differences in Salmonella serotype Typhimurium do not affect outcome of human disease.
\end{abstract}

\section{Background}

Globally, Salmonella enterica subsp. enterica is one of the leading food-borne pathogens. For example in 2006 in the United States, Salmonella enterica subsp. enterica caused 45.808 registered cases of salmonellosis, corresponding to an incidence of 15 cases/100,000 inhabitants [1]. Furthermore the actual number of infections is estimated to be 38 times higher [2]. In Denmark, there were 1658 registered cases of salmonellosis (incidence of 30 cases/100,000 inhabitants) in 2006 [3]. Salmonella serotype Typhimurium, denoted $S$. Typhimurium,

\footnotetext{
* Correspondence: evl@ssi.dk

${ }^{1}$ Statens Serum Institut, Bacterial Typing, Artillerivej 5, DK-2300 Copenhagen S, Denmark
}

accounted for $17 \%$ of the salmonellosis cases in the USA and $25 \%$ of the Danish cases [1,3]. The outcome of human infection ranges from mild self-limiting diarrhoea to severe diarrhoea that requires hospitalization. In rare cases, often among immunocompromised patients, salmonellosis can be fatal.

Several factors in both the host and the bacteria influence the outcome of an infection. Clearly an important aspect of human infection is the immune state of the patient. It has been shown that immunocompromised patients are more prone to develop a severe infection [4]. Another important aspect of human infection is the intestinal microbiota of the host. Ingestion of antibiotics is known to affect the intestinal microbiota leaving the 
host more prone to infection and disease caused by $S$. Typhimurium [5].

Significant bacterial factors for the outcome of infection are encoded by a wide range of genetic elements, including plasmids, prophages and Salmonella Pathogenicity Islands (SPIs). A total of 14 SPIs have been described so far [6]. SPI-1 encodes type 3 secretion system 1 (T3SS-1) that causes secretion and translocation of a range of bacterial proteins to the host cell. SPI-2 encodes T3SS-2 that allows intracellular survival and replication [7].

Different $S$. Typhimurium strains share more than 99\% genomic content [8]. The detected variation within $S$. Typhimurium is primarily represented by the prophages in the genome [9]. Variation in phenotype has also been demonstrated as there are different phagetypes of $S$. Typhimurium strains, and some of them can even show a high degree of variation in host adaptation [10]. Intra-serotype variability is also caused by the plasmids carried by $S$. Typhimurium, in particular, the Salmonella Virulence Plasmid (pSLT) which was observed more frequently in the strains isolated from blood than the strains isolated from faeces [11]. It has been proposed that this plasmid is significant in the spreading of an infectious strain from the intestine [12].

The recent development of microarray technology has allowed an extensive screening of many $S$. Typhimurium strains [13-15], but to our knowledge, no study has been able to link the molecular data obtained by microarray analysis of the strains to detailed epidemiological and clinical patient data.

We analyzed a collection of $S$. Typhimurium strains by DNA microarray analysis. These strains were selected on the basis of a previous epidemiological study where clinical data were obtained by means of patient interviews. The strains were selected from patients with mild infections and from patients with severe infections, and clinical data allowed us to correct for known underlying diseases and patient age. Strains were analyzed for presence or absence of 281 genes covering marker groups of genes related to pathogenicity, phages, antimicrobial resistance, fimbriae, mobility, serotype, and metabolism. We show that $S$. Typhimurium strains causing very different symptoms in patients had little genomic variation, and the observed variation does not correlate to the severity of disease.

\section{Results}

\section{Subtyping}

All strains were subtyped by Pulsed-field gel electrophoresis (PFGE), Multiple-locus variable-number of tandem-repeat analysis (MLVA) and Multilocus sequence typing (MLST). In general, the PFGE types of the strains correspond to the phagetype. All of the phagetype DT12 strains had the PFGE 22 profile and five out of six DT104 strains had the PFGE 14 profile. The remaining phagetypes showed different PFGE profiles (see additional file 1: $X b a$ I PFGE profiles of all isolates).

The MLVA types of the strains were all different. Loci STTR-9 and STTR-3 were the most conserved alleles and they had 1, 2 or 3 repeat units. STTR-5, STTR- 6 and STTR-10 were all alleles with varying repeat units. Some strains did not contain the STTR-10 allele at all, corresponding well to the fact that these strains were not carrying the pSLT (see additional file 2: Typing results of all strains).

The Sequence types (ST) of the strains were primarily ST19. Only three strains had other STs and these were ST376, ST35 and ST34 (see additional file 2: Typing results of all strains).

\section{DNA microarray marker groups Resistance and Serotyping}

The DNA microarray included 49 probes that targeted 10 different resistance genes and some of their known variants. The phenotypic resistance profiles all corresponded to the results obtained by the array (see additional file 3: Microarray results of all markers). The DNA microarray included 33 probes that target different $\mathrm{O}$-serogroups and $\mathrm{H}$-antigens. The $S$. Typhimurium serotype was confirmed by the array (see additional file 3 : Microarray results of all markers).

\section{DNA microarray marker groups Metabolism, Prophage and Mobility}

The DNA microarray contained 20 probes targeting different genes in the metabolism marker group. All strains on the array possessed 18 of the 20 metabolism genes. The gene SEN4287, which has been observed in serotypes $S$. Enteritidis, $S$. Dublin and $S$. Gallinarum and the gene STY4221, which has been found in serotypes $S$. Typhi and $S$. Paratyphi A, were not detected in any of the $S$. Typhimurium strains.

The DNA microarray contained 10 probes targeting different genes in the prophage marker group. Prophage related genes showed different present/absent profiles in different strains. All DT104 strains displayed identical profiles within the prophage marker group. Only DT104 strains possessed the ORF84 marker. The prophage genes $s b 10$ and $s b 54$ were present in all DT104 strains and some other strains. The genes STY3672, STY3676 and STY4625 were present in both DT10 strains and one of three RDNC strains. The remaining genes in the prophage marker group were identically present/absent in all strains (see additional file 3: Microarray results of all markers).

The DNA microarray contained 57 probes targeting different genes in the mobility marker group which also 
included plasmid incompatibility markers and IS-element markers. The markers showed high variability in the present/absent pattern among all strains. The variability correlated well with known properties of strains, e.g. all strains lacking the pSLT, also lacked the gene encoding the typical FIIA replicon of pSLT, and these strains also lacked the traT gene that is encoded in pSLT (see additional file 3: Microarray results of all markers).

\section{DNA microarray marker groups Pathogenicity and Fimbrial}

The pathogenicity marker group consisted of 87 markers and the strains had a highly similar pattern of present/absent genes within this marker group. Only the DT104 strains showed variation, as they lacked the gipA gene encoded by the Gifsy-1 prophage but harboured the two other genes encoded by Gifsy-1. The DT104 strains also possessed two other prophage-related genes which no other strains possessed. All strains harboured prophage Gifsy-2 and lacked prophages Gifsy-3 and Fels-1. Five strains of five different phagetypes lacked the pSLT and, therefore, lacked the virulence genes encoded in the plasmid. In total, $72 \%$ of the strains in this study carried the pSLT. The same percentage is observed when comparing all of the $S$. Typhimurium strains detected in Denmark between 2005 and 2009 (data not shown).

The SPI-1 to SPI-5 were present and SPI-7 was absent in all strains (Fig. 1).

The DNA microarray contained 22 probes targeting different genes in the fimbrial marker group. All strains showed identical patterns within this marker group, except for the pefA gene which is encoded in the pSLT. One strain carrying the pSLT did not show a positive reaction in the pefA probe (Fig. 1).

\section{Clustering of strains}

The microarray analysis clustered the strains into four major branches in a dendrogram (Fig. 2). The dendrogram is calculated from all markers except the resistance and serotyping markers as these could create a bias in the analysis. Cluster A had a depth of $96.1 \%$ and contained most of the DT12 strains but also other phagetypes. The strains in cluster A all harboured the pSLT, and all seven strains were fully sensitive to antimicrobial agents (see additional file 2: Typing results of all strains). In cluster A, two strains represented severe infection, four strains represented mild infection, and there was one outbreak strain. Cluster B had a depth of 98.6\% and contained all six DT104 strains, which all harboured the pSLT. Two of the DT104 strains were fully susceptible to antimicrobial agents. In cluster B, two strains represented severe infection, two strains

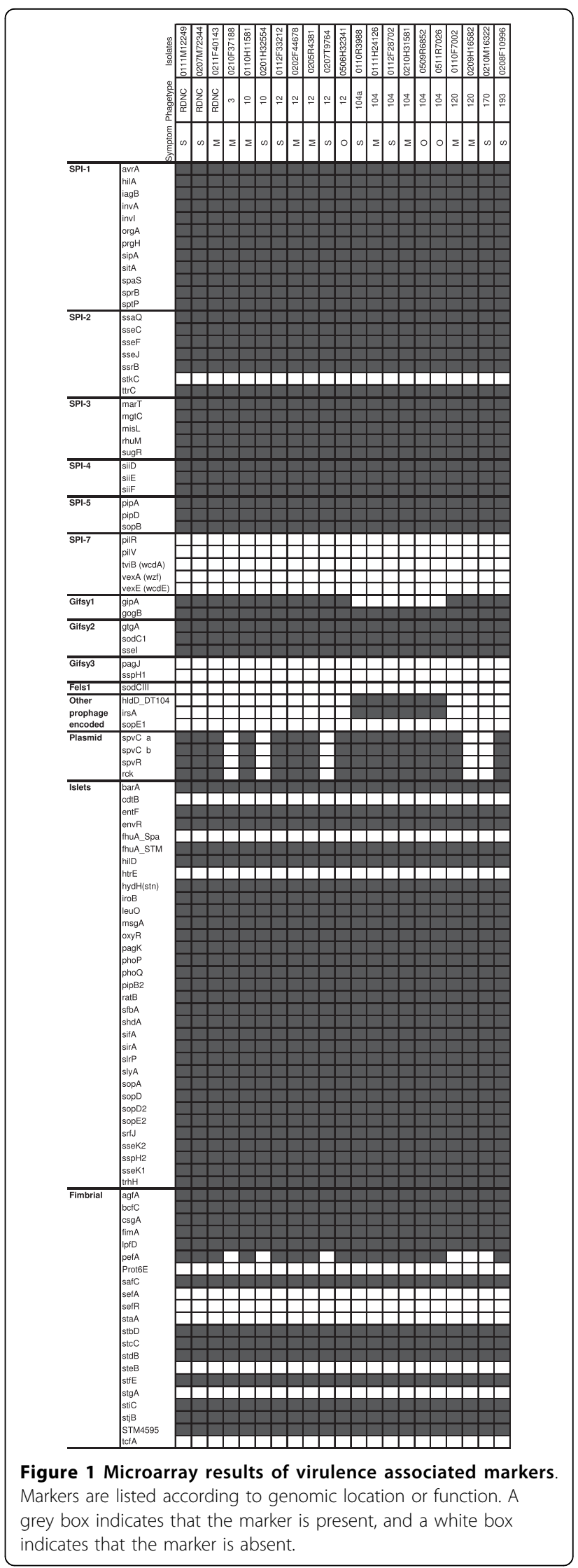




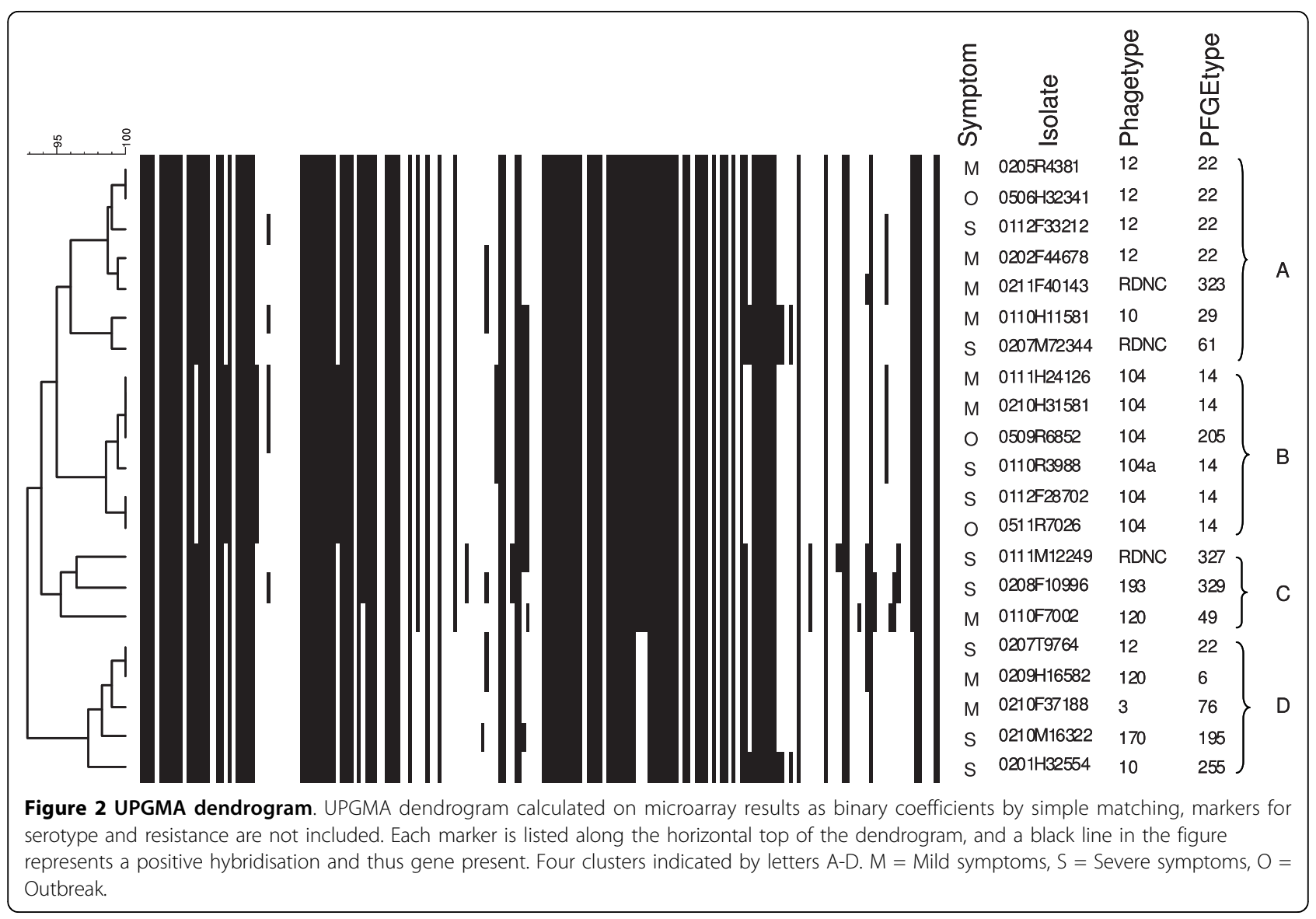

represented mild infection, and additionally there were two outbreak strains.

Cluster C had a depth of $95.2 \%$ and contained only three strains of three different phagetypes. All of the three strains carried the pSLT and showed resistance to at least four antimicrobial agents. The strains in cluster $\mathrm{C}$ branch off separately as they possess more genes from the mobility marker group which includes transposases. In cluster $C$, two strains represented severe infection and one strain represented mild infection. Cluster D had a depth of $97.2 \%$ and contained five strains of different phagetypes, including a DT12 strain, but none of the strains harboured the pSLT. One strain in cluster D showed resistance to three antimicrobial agents. In cluster D, three strains represented severe infection while two strains represented mild infection. In conclusion, strains causing severe and mild infection were represented equally across the dendrogram (Fig. 2).

\section{Discussion}

A collection of $S$. Typhimurium strains were analyzed and compared by the use of a microarray designed for characterization of Salmonella. The array consisted of 281 markers covering marker groups of genes related to pathogenicity, phages, antimicrobial resistance, fimbriae, mobility, serotype, and metabolism. Extensive background knowledge of patients regarding symptoms and underlying diseases enabled us to compare strains causing mild symptoms to strains causing severe symptoms.

Initial subtyping of the strains was performed using MLST and MLVA. These two methods target different parts of the chromosome and areas with different genetic variability, leading to differences in the discriminatory power of the methods. MLVA methods are generally high-discriminatory typing methods developed for outbreak investigations, and the $S$. Typhimurium MLVA method used in this study was specifically developed to differentiate the highly similar DT104 clone [16]. This method is based on highly variable repeat regions on the chromosome and on a plasmid. MLST is developed to estimate long term development and is based on conserved housekeeping genes with minimal variation [17]. Most strains in this study belonged to ST19, except for three strains with different STs. The difference between ST19 and each of the other STs was a single nucleotide change in one allele, so the similarity of the strains was high as expected with MLST. The strains all had different MLVA profiles, corresponding well with the fact 
that MLVA is a more discriminatory typing method and the strains were selected to be epidemiologically unrelated.

The strains were tested for antimicrobial resistance and eight of 21 strains showed resistance to three or more antimicrobial agents. Three strains from the group with mild symptoms, four strains from the group with severe symptoms, and a single outbreak strain showed resistance to antimicrobial agents. The resistance pattern did not correlate with the severity of disease in patients. The lack of increased virulence of resistant strains has previously been shown in DT104 strains $[18,19]$. An American study described that humans who ingested antimicrobials are more prone to get a subsequent infection with a resistant $S$. Typhimurium strain [20]. Two of the patients included in our study were administered antimicrobials within a month before onset of the $S$. Typhimurium infection, one patient was in the group with severe symptoms and the other patient was in the group with mild symptoms. Both infections were caused by resistant $S$. Typhimurium strains.

Differences between the $S$. Typhimurium strains were detected in the prophage marker group. The DT104 strains were different as seen by detection of the ORF84 marker, previously shown to be present primarily in DT104 strains [21]. The Salmonella prophage ST64B (sb10 and sb54) was detected in different phagetypes in this study, but notably this prophage is present in all DT104 strains, and these observations corresponded to previous findings [22]. Other genes showing variability within the prophage marker group were the $S$. Typhi specific genes STY3672, STY3676 and STY4625. The markers of these genes were detected in three $S$. Typhimurium strains. The occurrence of these genes could be explained by lacking specificity of the probe, or by point mutations in the $S$. Typhimurium strains leading to cross-hybridization. Prophages are known to contribute to virulence in mice [23] but presence or absence of prophages does not correlate with any differences in symptoms caused by strains in our study investigating strains isolated from humans.

The mobility marker group also displayed variation between strains, but most variation related to incompatibility groups of plasmids and probes encoding transposons. The variation did not correspond to any phagetypes or disease symptoms.

The strains showed highly similar profiles when comparing the virulence associated genes. Some variation was detected between other phagetypes and the DT104 strains which were the only strains containing the hldD gene and the irs $A$ gene, but these genes have previously been shown to be specific for the DT104 phagetype [24]. Also the Gifsy-1 encoded genes showed variation between other phagetypes and the DT104 strains, as the
DT104 strains lacked one of three Gifsy-1 encoded genes present on the array. The gene lacking in our DT104 strains is consistent with an observation made recently in a study comparing the genome sequence of a DT104 strain to a $S$. Typhimurium LT2 strain [25]. The study observes a prophage sequence in DT104 which only shows partly homology to the Gifsy-1 prophage sequence. All other strains in our study possessed the Gifsy-1 prophage. The SPI-1 to SPI-5 were present in all strains but the SPI-7 was absent. SPI-7 was initially reported in a $S$. Typhi [26], and similar islands were detected in $S$. Dublin and $S$. Paratyphi C [27]. The pSLT is another important virulence marker. In an American study, pSLT was shown to be present in $76 \%$ of strains isolated from blood compared to $42 \%$ of strains isolated from faeces [11], however, in the present study the virulence plasmid was present in $72 \%$ of the strains, even though the strains were all isolated from faeces and some strains caused very mild disease symptoms. The selected $S$. Typhimurium strains are representative for the Danish $S$. Typhimurium population regarding the presence of $\mathrm{pSLT}$, as $72 \%$ of all Danish $S$. Typhimurium isolates from 2005 until 2009 carried the plasmid. Out of five strains lacking the pSLT, three had caused severe symptoms. Interestingly, strains can cause infection with severe symptoms even if they lack the plasmid. Furthermore, strains can carry the pSLT and only cause infection with mild symptoms. In this study, the presence or absence of pSLT did not correspond to any phagetypes or disease symptoms.

The dendrogram calculated on the basis of the array results showed clustering of the strains into four groups. The clustering confirmed DT104 as being a clonal phagetype, but a number of probes were also designed to target only DT104 strains, and that might emphasize the separate clustering of this phagetype. The clustering of the remaining groups was mainly correlated to the presence or absence of the pSLT and other mobile elements such as transposons. The four clusters in the tree represented an almost equal amount of strains causing severe or mild symptoms of $S$. Typhimurium infections.

The probes on the array were designed primarily on basis of the $S$. Typhimurium LT2 sequence, but also some additional known genes from other serotypes such as $S$. Enteritidis and $S$. Typhi. The presence or absence of additional $S$. Typhimurium genes, which are not present in the LT2 sequence, could not be assessed in this study. It is possible that the presence or absence of such genes, not present in LT2, are responsible for the observed differences in the patient symptoms. Although this is not likely, as recent publications of sequenced $S$. Typhimurium strains showed few gene differences to the LT2 sequenced strain $[28,29]$. 


\section{Conclusion}

We investigated a collection of Salmonella strains for the presence of a wide range of known virulence genes, and detected no significant difference in the presence of these genes. The investigated strains were carefully selected, based on epidemiological data, to represent strains causing severe symptoms of disease and strains causing mild symptoms of disease. Although the investigated strains had different genomic contents, this study found no evidence of a correlation between the genomic contents of the $S$. Typhimurium strains and the symptoms they caused in human cases of salmonellosis. Based on the results of this study, an idea which immediately suggests itself is that the factors and defence mechanisms of the host immune system may play a fundamental role in the different outcomes of infection.

\section{Methods}

\section{Patient interviews}

Data for the present study was obtained from a prospective cohort study carried out in Denmark from September 2001 to December 2002 [30]. Cases were patients with a culture-confirmed $S$. Typhimurium infection, identified by the examination of samples submitted to Statens Serum Institut (SSI) from hospitals and general practitioners. Patients were invited to participate by their own physicians or the relevant hospital department. Individuals who agreed to participate were mailed a questionnaire and asked to complete the questionnaire immediately. Data was collected by a computer-assisted telephone interviewing system (CATI) whilst the subjects were looking at their questionnaire. This method facilitated data collection and allowed standardized probing about relevant exposures and outcomes. Data collected included information on clinical symptoms, treatment, medications (including antimicrobials) from one month before infection to one month after, underlying illnesses, foreign travel during the two weeks prior to inclusion and basic socioeconomic variables i.e. education, occupation and household income. Cases were excluded for the following reasons: an earlier history of Salmonella infection within six month prior to onset of the current infection, lack of communication skills in Danish, or if the patient's health care provider deemed the patient unable to complete interview due to an impaired mental state. A parent was interviewed if the patient was under 15 years of age and if the patients were between 15 and 18 years of age they could be interviewed - subject to parental approval. This study was reported to The Danish Data Protection Agency and has been approved by the regional scientific ethical committee of Copenhagen and Frederiksberg Municipality (KEF 01-031/01).

\section{Selection of strains}

Faecal strains of $S$. Typhimurium were chosen based on the previously described patient interviews. Strains from patients over the age of 65 years and strains from patients with known underlying diseases were not included in this study. The patients were sorted according to hospitalization data and fever. Two groups were then established: a severe infection group with patients who were hospitalized due to their $S$. Typhimurium infection and also had a fever; and a mild infection group with patients who were not hospitalized and did not have a fever. From each of these groups nine strains were selected, aiming to represent the same phagetypes in each group (Table 1). The phagetype distribution observed within all strains from the interviewed patients, not including the patients with known underlying disease, correlated to the overall distribution of all human S. Typhimurium strains in Denmark in 2001 and 2002. A pattern of specific phagetypes relating to specific symptoms was not observed within the entire interview material (data not shown). Of the nine hospitalized patients, four had bloody stools. Furthermore, three outbreak strains were included in the study, representing strains with known high virulence potential. All faecal samples received at SSI in Denmark are screened for double infection with frequently occurring intestinal

Table 1 Isolate information and degree of disease symptoms.

\begin{tabular}{|c|c|c|c|c|}
\hline Isolate $\mathrm{nr}$ & $\begin{array}{l}\text { Phage } \\
\text { type }\end{array}$ & $\begin{array}{l}\text { Patient } \\
\text { age }\end{array}$ & $\begin{array}{c}\text { Year of } \\
\text { isolation }\end{array}$ & $\begin{array}{c}\text { Disease } \\
\text { symptoms }\end{array}$ \\
\hline 0210F37188 & 3 & 39 & 2002 & Mild \\
\hline $0110 \mathrm{H} 11581$ & 10 & 38 & 2001 & Mild \\
\hline $0202 F 44678$ & 12 & 30 & 2002 & Mild \\
\hline 0205R4381 & 12 & 41 & 2002 & Mild \\
\hline $0111 \mathrm{H} 24126$ & 104 & 62 & 2001 & Mild \\
\hline $0210 \mathrm{H} 31581$ & 104 & 14 & 2002 & Mild \\
\hline $0110 F 7002$ & 120 & 63 & 2001 & Mild \\
\hline 0209H16582 & 120 & 0 & 2002 & Mild \\
\hline $0211 F 40143$ & RDNC & 1 & 2002 & Mild \\
\hline $0201 \mathrm{H} 32554$ & 10 & 3 & 2002 & Severe \\
\hline 0112 F33212 & 12 & 47 & 2001 & Severe \\
\hline 0207T9764 & 12 & 11 & 2002 & Severe \\
\hline $0112 F 28702$ & 104 & 20 & 2001 & Severe \\
\hline $0110 R 3988$ & $104 a$ & 36 & 2001 & Severe \\
\hline 0210M16322 & 170 & 19 & 2002 & Severe \\
\hline 0208F10996 & 193 & 9 & 2002 & Severe \\
\hline $0111 M 12249$ & RDNC & 12 & 2001 & Severe \\
\hline 0207M72344 & RDNC & 16 & 2002 & Severe \\
\hline $0506 \mathrm{H} 32341$ & 12 & 53 & 2005 & Outbreak \\
\hline 0509R6852 & 104 & 58 & 2005 & Outbreak \\
\hline $0511 R 7026$ & 104 & 2 & 2005 & Outbreak \\
\hline
\end{tabular}


pathogens such as Campylobacter, Shigella, Yersinia and others. All strains used in this study were confirmed as originating from a single-organism infection.

\section{Serotyping}

All strains were previously serotyped at SSI according to the White-Kauffmann-Le Minor scheme [31] by agglutination with $\mathrm{O}$ - and $\mathrm{H}$-antigen specific sera (SSI Diagnostika, Hillerød, Denmark). All strains belonged to Salmonella enterica serotype Typhimurium.

\section{Antimicrobial susceptibility testing}

Susceptibility to a standard panel of antimicrobial agents http://www.danmap.org was determined by microbroth dilution and interpreted according to Clinical and Laboratory Standards Institute guidelines [32], except for ciprofloxacin $(\geq 0.125 \mu \mathrm{g} / \mathrm{mL}$ was used as breakpoint).

\section{Phage typing}

Phage typing was performed by the National Food Institute, Technical University of Denmark according to the phage typing scheme developed by Callow [33] and extended by Anderson et al. [34]. Strains that react with phages, but have a profile not included in the phage typing scheme, are denoted Reaction Does Not Conform (RDNC).

\section{DNA microarray}

The DNA microarray used in this study was previously described [35]. A set of 281 gene-specific 57-60 mer oligonucleotide probes were designed using the program Array Designer 4.1 (Premier Biosoft, Palo Alto, CA, USA). The oligonucleotides were spotted on glass slides using a QArray Mini Arrayer (Genetix, New Milton, UK). Hybridized spots were visualized in a GenePix 4000B laser scanner (Axon, Foster City, CA). Each oligonucleotide allows the detection of the presence or absence of a characteristic sequence previously described in Salmonella. The microarray used gives no information on the location of a gene or target sequence and can only score its presence or absence. Uncertain array results were resolved by PCR using primers described previously [35].

\section{Data analysis}

Analysis of the DNA microarray data was performed as previously described [35]. A comparison was made by importing array values, gene present or absent, into BioNumerics 5.1 (Applied Maths, Sint-Martens-Latem, Belgium) as character data. An Unweighted Pair Group Method with Arithmetic mean (UPGMA) dendrogram (Fig. 2) was calculated by simple matching of binary coefficients on the basis of a geneset consisting of all genes from the array except the serotyping markers and the resistance markers.

\section{Multiple-locus variable-number of tandem-repeat analysis (MLVA)}

MLVA was performed as described previously [36] to ensure that the strains were likely to be epidemiologically unrelated. The MLVA repeats were calculated and named according to the method described recently [37].

\section{Pulsed-field gel electrophoresis (PFGE)}

PFGE was carried out with $\mathrm{XbaI}$ restriction enzyme according to the Pulse-Net protocol [38], gels were analyzed in BioNumerics 5.1, and profiles were assigned by comparison to a database with known Danish profiles (see additional file 1: Xba I PFGE profiles of all isolates).

\section{Sequence typing}

Multilocus sequence typing (MLST) was carried out as previously described [39] and the alleles and the sequence types were assigned according to the MLST scheme on http://mlst.ucc.ie/mlst/mlst/dbs/Senterica/.

Additional file 1: PFGE profiles. Xba I PFGE profiles of all isolates

Additional file 2: Typing results of all strains

Additional file 3: Microarray results of all markers. Markers are listed alphabetically within marker groups. A grey box indicates the marker being present and a white box indicates the marker being absent.

\section{Acknowledgements}

EL was partly funded by the Danish Research Council. We are grateful to the patients without whose kind participation this study would not have been possible. Written consent was obtained from all patients or in case of minors from their parents before participating in the study.

\section{Author details}

'Statens Serum Institut, Bacterial Typing, Artillerivej 5, DK-2300 Copenhagen S, Denmark. ${ }^{2}$ Federal Institute for Risk Assessment, National Reference Laboratory for Salmonella, Diedersdorfer Weg 1, D-12277 Berlin, Germany. ${ }^{3}$ Department of Internal Medicine, Roskilde Sygehus, 4000 Roskilde, Denmark. ${ }^{4}$ University of Copenhagen, Faculty of Life Sciences, Bülowsvej 17, DK-1870 Frederiksberg C, Denmark.

\section{Authors' contributions}

EL carried out the microarray experiments, the MLST analysis, participated in the study design and drafted the manuscript. MT participated in conceiving and designing the study. BM designed the microarray. SH participated in the microarray experiments and participated in drafting the Methods section. $\mathrm{MH}$ carried out the patient interviews and the epidemiological analysis and participated in drafting the Methods section. HC participated in conceiving and designing the study. EMN participated in conceiving and designing the study. All authors read and approved the final manuscript.

Received: 14 October 2009 Accepted: 31 March 2010

Published: 31 March 2010

\section{References}

1. McNabb SJ, Jajosky RA, Hall-Baker PA, Adams DA, Sharp P, Worshams C, Anderson WJ, Javier AJ, Jones GJ, Nitschke DA, et al: Summary of notifiable diseases-United States, 2006. MMWR Morb Mortal Wkly Rep 2008, 55:1-92. 
2. Voetsch AC, Van Gilder TJ, Angulo FJ, Farley MM, Shallow S, Marcus R, Cieslak PR, Deneen VC, Tauxe RV: FoodNet estimate of the burden of illness caused by nontyphoidal Salmonella infections in the United States. Clin Infect Dis 2004, 38(Suppl 3):S127-S134.

3. Anonymous: Annual Report on Zoonoses in Denmark 20062006.

4. Gordon MA: Salmonella infections in immunocompromised adults. $J$ Infect 2008, 56:413-422.

5. Lawley TD, Bouley DM, Hoy YE, Gerke C, Relman DA, Monack DM: Host transmission of Salmonella enterica serovar Typhimurium is controlled by virulence factors and indigenous intestinal microbiota. Infect Immun 2008, 76:403-416.

6. Morgan E: Salmonella Pathogenicity Islands. Salmonella Molecular Biology and Pathogenesis Horizon BioscienceRhen M, Maskell D, Mastroeni P, Threlfall EJ 2007.

7. Layton AN, Galyov EE: Salmonella -induced enteritis: molecular pathogenesis and therapeutic implications. Expert Rev Mol Med 2007, 9:1-17.

8. Chan K, Baker S, Kim CC, Detweiler CS, Dougan G, Falkow S: Genomic comparison of Salmonella enterica serovars and Salmonella bongori by use of an S. enterica serovar Typhimurium DNA microarray. J Bacteriol 2003, 185:553-563.

9. Drahovska H, Mikasova E, Szemes T, Ficek A, Sasik M, Majtan V, Turna J: Variability in occurrence of multiple prophage genes in Salmonella Typhimurium strains isolated in Slovak Republic. FEMS Microbiol Lett 2007 270:237-244.

10. Rabsch W, Andrews HL, Kingsley RA, Prager R, Tschape H, Adams LG, Baumler AJ: Salmonella enterica serotype Typhimurium and its hostadapted variants. Infect Immun 2002, 70:2249-2255.

11. Fierer J, Krause M, Tauxe R, Guiney D: Salmonella typhimurium bacteremia: association with the virulence plasmid. J Infect Dis 1992, 166:639-642.

12. Fierer J, Guiney DG: Diverse virulence traits underlying different clinical outcomes of Salmonella infection. J Clin Invest 2001, 107:775-780.

13. Malorny B, Bunge C, Guerra B, Prietz S, Helmuth R: Molecular characterisation of Salmonella strains by an oligonucleotide multiprobe microarray. Mol Cell Probes 2007, 21:56-65.

14. Scaria J, Palaniappan RU, Chiu D, Phan JA, Ponnala L, McDonough P, Grohn YT, Porwollik S, McClelland M, Chiou CS, et al: Microarray for molecular typing of Salmonella enterica serovars. Mol Cell Probes 2008, 22:238-243.

15. Porwollik S, Boyd EF, Choy C, Cheng P, Florea L, Proctor E, McClelland M: Characterization of Salmonella enterica subspecies I genovars by use of microarrays. J Bacteriol 2004, 186:5883-5898.

16. Lindstedt BA, Heir E, Gjernes E, Kapperud G: DNA fingerprinting of Salmonella enterica subsp. enterica serovar Typhimurium with emphasis on phage type DT104 based on variable number of tandem repeat loci. J Clin Microbiol 2003, 41:1469-1479.

17. Enright MC, Spratt BG: Multilocus sequence typing. Trends Microbiol 1999, 7:482-487.

18. Threlfall EJ, Ward LR, Rowe B: Multiresistant Salmonella Typhimurium DT 104 and Salmonella bacteraemia. Lancet 1998, 352:287-288.

19. Carlson SA, Browning M, Ferris KE, Jones BD: Identification of diminished tissue culture invasiveness among multiple antibiotic resistant Salmonella Typhimurium DT104. Microb Pathog 2000, 28:37-44.

20. Glynn MK, Reddy V, Hutwagner L, Rabatsky-Ehr T, Shiferaw B, Vugia DJ, Segler S, Bender J, Barrett TJ, Angulo FJ: Prior antimicrobial agent use increases the risk of sporadic infections with multidrug-resistant Salmonella enterica serotype Typhimurium: a FoodNet case-control study, 1996-1997. Clin Infect Dis 2004, 38(Suppl 3):S227-S236.

21. Malorny B, Schroeter A, Bunge C, Helmuth R: Prevalence of Escherichia coli 0157:H7 prophage-like sequences among German Salmonella enterica serotype Typhimurium phage types and their use in detection of phage type DT104 by the polymerase chain reaction. Vet Microbiol 2002, 87:253-265.

22. Hermans AP, Beuling AM, van Hoek AH, Aarts $H J$, Abee T, Zwietering MH: Distribution of prophages and SGI-1 antibiotic-resistance genes among different Salmonella enterica serovar Typhimurium isolates. Microbiology 2006, 152:2137-2147.

23. Figueroa-Bossi N, Uzzau S, Maloriol D, Bossi L: Variable assortment of prophages provides a transferable repertoire of pathogenic determinants in Salmonella. Mol Microbiol 2001, 39:260-271.
24. Hermans AP, Abee T, Zwietering MH, Aarts HJ: Identification of novel Salmonella enterica serovar Typhimurium DT104-specific prophage and nonprophage chromosomal sequences among serovar Typhimurium isolates by genomic subtractive hybridization. Appl Environ Microbiol 2005, 71:4979-4985

25. Cooke FJ, Brown DJ, Fookes M, Pickard D, Ivens A, Wain J, Roberts M, Kingsley RA, Thomson NR, Dougan G: Characterisation of the genomes of a diverse collection of Salmonella enterica serovar Typhimurium definitive phage type 104 (DT104). J Bacteriol 2008, 190:8155-8162.

26. Parkhill J, Dougan G, James KD, Thomson NR, Pickard D, Wain J, Churcher C, Mungall KL, Bentley SD, Holden MT, et al: Complete genome sequence of a multiple drug resistant Salmonella enterica serovar Typhi CT18. Nature 2001, 413:848-852.

27. Pickard D, Wain J, Baker S, Line A, Chohan S, Fookes M, Barron A, Gaora PO, Chabalgoity JA, Thanky N, et al: Composition, acquisition, and distribution of the Vi exopolysaccharide-encoding Salmonella enterica pathogenicity island SPI-7. J Bacteriol 2003, 185:5055-5065.

28. Jarvik T, Smillie C, Groisman EA, Ochman H: Short-term Signatures of Evolutionary Change in the Salmonella enterica serovar Typhimurium 14028 Genome. J Bacteriol 2009, 192:560-567.

29. Kingsley RA, Msefula CL, Thomson NR, Kariuki S, Holt KE, Gordon MA, Harris D, Clarke L, Whitehead S, Sangal V, et al: Epidemic multiple drug resistant Salmonella Typhimurium causing invasive disease in subSaharan Africa have a distinct genotype. Genome Res 2009, 19:2279-2287.

30. Helms M: Health impact of zoonotic Salmonella and other foodborne bacterial gastrointestinal infections, with particular reference to antimicrobial drug resistance in Salmonella Typhimurium. PhD Thesis Danish Epidemiology Science Centre, Statens Serum Institut 2005.

31. Grimont PA, Weill FX: Antigenic formulae of the Salmonella serovars 2007.

32. Wayne PA: Clinical and Laboratory Standards Institute. Performance standards for antimicrobial susceptility testing, 18th international supplement. CLSI document M100-S18. Wayne, PA: CLSI; 2008. CLSI 2008.

33. Callow BR: A new phage-typing scheme for Salmonella typhi-murium. J Hyg (Lond) 1959, 57:346-359.

34. Anderson ES, Ward LR, De Saxe MJ, de Sa JDH: Bacteriophage-typing designations of Salmonella typhimurium. J Hyg (Lond) 1977, 78:297-300.

35. Huehn S, Bunge C, Junker E, Helmuth R, Malorny B: Poultry-associated Salmonella enterica subsp. enterica serovar 4,12:d:- reveals high clonality and a distinct pathogenicity gene repertoire. Appl Environ Microbiol 2009, 75:1011-1020.

36. Torpdahl M, Sorensen G, Lindstedt BA, Nielsen EM: Tandem repeat analysis for surveillance of human Salmonella Typhimurium infections. Emerg Infect Dis 2007, 13:388-395.

37. Larsson J, Torpdahl M, Petersen RF, Sørensen G, Lindstedt BA, Nielsen EM: Development of a new nomenclature for Salmonella Typhimurium multi-locus tandem repeats analysis (MLVA). Euro Surveill 2009, 14:pii, 19174.

38. Ribot EM, Fair MA, Gautom R, Cameron DN, Hunter SB, Swaminathan B, Barrett TJ: Standardization of pulsed-field gel electrophoresis protocols for the subtyping of Escherichia coli 0157:H7, Salmonella, and Shigella for PulseNet. Foodborne Pathog Dis 2006, 3:59-67.

39. Kidgell C, Reichard U, Wain J, Linz B, Torpdahl M, Dougan G, Achtman M: Salmonella Typhi, the causative agent of typhoid fever, is approximately 50,000 years old. Infect Genet Evol 2002, 2:39-45.

doi:10.1186/1471-2180-10-96

Cite this article as: Litrup et al:: DNA microarray analysis of Salmonella serotype Typhimurium strains causing different symptoms of disease. BMC Microbiology 2010 10:96. 